\title{
Affine Moment Invariants of Color Images ${ }^{\star}$
}

\author{
Tomáš Suk and Jan Flusser \\ Institute of Information Theory and Automation, \\ Academy of Sciences of the Czech Republic, \\ Pod vodárenskou věží 4, 18208 Prague 8, Czech Republic \\ $\{$ flusser, suk\}@utia.cas.cz \\ http://www.utia.cas.cz
}

\begin{abstract}
A new type of affine moment invariants for color images is proposed in this paper. The traditional affine moment invariants can be computed on each color channel separately, yet when the channels are transformed together, by the same affine transform, additional invariants can be computed. They have low order and therefore high robustness to noise. The new invariants are compared with another set of invariants for color images using second powers of the image function. The basic properties of the new features are tested on real images in a numerical experiment.
\end{abstract}

\section{Introduction}

The pattern recognition of objects on images distorted by affine transform has been studied for many years. Affine moment invariants showed suitability for this purpose, however they are typically computed from binary or gray-level images. In case of color images, additional features can be computed using color.

Certain satellites are capable of capturing a large number (let us say $n$ ) of spectral bands (often infrared, less commonly ultraviolet or visible), the image from one spectral band is called channel. Ordinary color photographs are much more readily accessible, and since they contain 3 visible spectral bands they can be considered multi-channel images with $n=3$. Typically, the color is used for better segmentation and invariants are computed from binary silhouettes of the segmented objects, in certain cases the first principal component with zeroed background is used.

In pattern recognition the following rule applies and must be satisfied: the number $i$ of independent invariants equals the number $m$ of independent measurements of a certain object (the number of moments in our case) minus the number $t$ of independent constraints(see e.g. [1]). In most cases it equals the number of parameters of the transformation, in case of the affine transform, $t=6$, thus this rule can be rewritten as $i=m-t$.

If we decide to compute the invariants directly from channels, we can use each channel separately and obtain triple ( $n$-fold) features. Nevertheless, if we use $m$

\footnotetext{
* This work has been supported by the grant No. 102/08/1593 of the Grant Agency of the Czech Republic.
} 
moments of each $n$ channels and $t$ parameters of the transform, then we obtain $i=n(m-t)$ independent invariants of separate channels.

In case of multi-channel images, where there is no geometric transform between channels, we can suppose a two-dimensional affine transform with identical parameters in each channel. In this case, we could obtain $n m-t$ independent invariants, and thus $t(n-1)$ additional invariants, $t$ for each additional channel.

Mindru et al published a series of conference contributions, e.g. 22 and 3] including a survey in the journal paper 4] with the combined invariants to the affine transform and illumination changes, where moments computed from a certain power of the channels were utilized. Their generalized color moment of order $s=p+q$ and degree $d=\alpha+\beta+\gamma$ of a certain object $\Omega$ is then defined

$$
M_{p q}^{\alpha \beta \gamma}=\iint_{\Omega} x^{p} y^{q}(R(x, y))^{\alpha}(G(x, y))^{\beta}(B(x, y))^{\gamma} d x d y
$$

where $R, G$, and $B$ are three color channels. The authors use these moments for the construction of combined invariants to the affine transform of coordinates and contrast changes. In their most complex version, they suppose a general affine transform of $R G B$ values.

In the case of an infinite set of moments of all orders, only the moments where $d=1$ are independent, e.g. if we know $M_{p q}^{\alpha \beta \gamma}$ for $s=0$ and $\alpha, \beta, \gamma=$ $0,1, \ldots, 255$, we could theoretically reconstruct the complete $3 \mathrm{D}$ histogram. This redundancy decreases as the maximum order of moments decreases, for loworder moments this method may yield meaningful results. However, even for low orders, using higher powers of brightness in individual channels is more sensitive to nonlinearity of the contrast changes and may lead to misclassification.

The problematic issue in certain applications is not illumination changes, but exclusively or almost exclusively the geometric distortion. In such cases using traditional moments with $d=1$ is more suitable and construction of a commensurate feature set is the theme of this contribution.

\section{Moment Invariants of Multi-channel Images}

Affine transformation is a general linear transform of space coordinates of an image. It can be expressed as

$$
\begin{aligned}
& u=a_{0}+a_{1} x+a_{2} y \\
& v=b_{0}+b_{1} x+b_{2} y
\end{aligned}
$$

An exact model of photographing a planar scene by a pin-hole camera whose optical axis is not perpendicular to the scene is a projective transform. Since the projective transform is not linear, its Jacobian is a function of spatial coordinates and projective moment invariants from a finite number of moments cannot exist. The perspective effect is negligible for small objects and large camera-to-scene distances and thus the affine transform can be used as good approximation of the projective transform. 
The geometric moment $m_{p q}$ of the order $s=p+q$ of an integrable image function $f(x, y)$ is defined as

$$
m_{p q}^{(f)}=\int_{-\infty}^{\infty} \int_{-\infty}^{\infty} x^{p} y^{q} f(x, y) d x d y
$$

If the coordinates are translated so their origin appears the centroid of the image $x_{c}^{(f)}=m_{10}^{(f)} / m_{00}^{(f)}, \quad y_{c}^{(f)}=m_{01}^{(f)} / m_{00}^{(f)}$, they are called central moments

$$
\mu_{p q}^{(f)}=\int_{-\infty}^{\infty} \int_{-\infty}^{\infty}\left(x-x_{c}\right)^{p}\left(y-y_{c}\right)^{q} f(x, y) d x d y
$$

The first few affine moment invariants, the derivation of which can be found in e.g. [5], 6], or [7], are as follows:

$$
\begin{aligned}
I_{1}= & \left(\mu_{20} \mu_{02}-\mu_{11}^{2}\right) / \mu_{00}^{4} \\
I_{2}= & \left(-\mu_{30}^{2} \mu_{03}^{2}+6 \mu_{30} \mu_{21} \mu_{12} \mu_{03}-4 \mu_{30} \mu_{12}^{3}-4 \mu_{21}^{3} \mu_{03}+3 \mu_{21}^{2} \mu_{12}^{2}\right) / \mu_{00}^{10} \\
I_{3}= & \left(\mu_{20} \mu_{21} \mu_{03}-\mu_{20} \mu_{12}^{2}-\mu_{11} \mu_{30} \mu_{03}+\mu_{11} \mu_{21} \mu_{12}+\mu_{02} \mu_{30} \mu_{12}-\mu_{02} \mu_{21}^{2}\right) / \mu_{00}^{7} \\
I_{4}= & \left(-\mu_{20}^{3} \mu_{03}^{2}+6 \mu_{20}^{2} \mu_{11} \mu_{12} \mu_{03}-3 \mu_{20}^{2} \mu_{02} \mu_{12}^{2}-6 \mu_{20} \mu_{11}^{2} \mu_{21} \mu_{03}-6 \mu_{20} \mu_{11}^{2} \mu_{12}^{2}\right. \\
& +12 \mu_{20} \mu_{11} \mu_{02} \mu_{21} \mu_{12}-3 \mu_{20} \mu_{02}^{2} \mu_{21}^{2}+2 \mu_{11}^{3} \mu_{30} \mu_{03}+6 \mu_{11}^{3} \mu_{21} \mu_{12} \\
& \left.-6 \mu_{11}^{2} \mu_{02} \mu_{30} \mu_{12}-6 \mu_{11}^{2} \mu_{02} \mu_{21}^{2}+6 \mu_{11} \mu_{02}^{2} \mu_{30} \mu_{21}-\mu_{02}^{3} \mu_{30}^{2}\right) / \mu_{00}^{11} .
\end{aligned}
$$

The index $(f)$ can be omitted, if the invariants are computed from one channel only. The theory of algebraic invariants (e.g. 8, among many others) offers using simultaneous invariants, i.e. invariants from moments of more than one order. They preserve their invariance even if we compute the moments of different orders on different objects, the only constraint is the common affine transform of the objects. We can choose two channels (let us label them $a$ and $b$ ), take an arbitrary simultaneous invariant, e.g. $I_{3}$, and substitute second-order moments computed on one channel and third-order moments computed on the other channel

$$
\begin{aligned}
I_{C 23}^{(a, b)}= & \left(\mu_{20}^{(a)} \mu_{21}^{(b)} \mu_{03}^{(b)}-\mu_{20}^{(a)}\left(\mu_{12}^{(b)}\right)^{2}-\mu_{11}^{(a)} \mu_{30}^{(b)} \mu_{03}^{(b)}+\mu_{11}^{(a)} \mu_{21}^{(b)} \mu_{12}^{(b)}+\mu_{02}^{(a)} \mu_{30}^{(b)} \mu_{12}^{(b)}-\right. \\
& \left.-\mu_{02}^{(a)}\left(\mu_{21}^{(b)}\right)^{2}\right) / \mu_{00}^{7}
\end{aligned}
$$

where $\mu_{00}=\mu_{00}^{(a)}+\mu_{00}^{(b)}$. The letter $C$ in the index represents a common invariant of more channels (or color invariant) and the numbers stand for orders of the moments. We can also utilize algebraic invariants of two or more binary forms with the same order. An example of such an invariant is of the second order

$$
I_{C 2}^{(a, b)}=\left(\mu_{20}^{(a)} \mu_{02}^{(b)}+\mu_{20}^{(b)} \mu_{02}^{(a)}-2 \mu_{11}^{(a)} \mu_{11}^{(b)}\right) / \mu_{00}^{4}
$$

If we use moments of one channel only (i.e. $a=b$ ), we obtain essentially $I_{1}$. Another such invariant is of the third order

$$
I_{C 3}^{(a, b)}=\left(\mu_{30}^{(a)} \mu_{03}^{(b)}-3 \mu_{21}^{(a)} \mu_{12}^{(b)}+3 \mu_{21}^{(b)} \mu_{12}^{(a)}-\mu_{30}^{(b)} \mu_{03}^{(a)}\right) / \mu_{00}^{5}
$$


If we use moments of one channel only, it becomes zero. The third-order invariant of degree two from one channel does not exist, while that from two channels does exist. An example of three-channel fourth-order symmetric invariant is as follows

$$
\begin{aligned}
& I_{C 4}^{(a, b, c)}=\left(\mu_{40}^{(a)} \mu_{22}^{(b)} \mu_{04}^{(c)}+\mu_{40}^{(a)} \mu_{22}^{(c)} \mu_{04}^{(b)}+\mu_{40}^{(b)} \mu_{22}^{(a)} \mu_{04}^{(c)}+\mu_{40}^{(b)} \mu_{22}^{(c)} \mu_{04}^{(a)}+\right. \\
& +\mu_{40}^{(c)} \mu_{22}^{(a)} \mu_{04}^{(b)}+\mu_{40}^{(c)} \mu_{22}^{(b)} \mu_{04}^{(a)}-2 \mu_{40}^{(a)} \mu_{13}^{(b)} \mu_{13}^{(c)}-2 \mu_{40}^{(b)} \mu_{13}^{(a)} \mu_{13}^{(c)}-2 \mu_{40}^{(c)} \mu_{13}^{(a)} \mu_{13}^{(b)}- \\
& -2 \mu_{31}^{(a)} \mu_{31}^{(b)} \mu_{04}^{(c)}-2 \mu_{31}^{(a)} \mu_{31}^{(c)} \mu_{04}^{(b)}-2 \mu_{31}^{(b)} \mu_{31}^{(c)} \mu_{04}^{(a)}+2 \mu_{31}^{(a)} \mu_{22}^{(b)} \mu_{13}^{(c)}+2 \mu_{31}^{(a)} \mu_{22}^{(c)} \mu_{13}^{(b)}+ \\
& +2 \mu_{31}^{(b)} \mu_{22}^{(a)} \mu_{13}^{(c)}+2 \mu_{31}^{(b)} \mu_{22}^{(c)} \mu_{13}^{(a)}+2 \mu_{31}^{(c)} \mu_{22}^{(a)} \mu_{13}^{(b)}+2 \mu_{31}^{(c)} \mu_{22}^{(b)} \mu_{13}^{(a)}- \\
& \left.-6 \mu_{22}^{(a)} \mu_{22}^{(b)} \mu_{22}^{(c)}\right) / \mu_{00}^{9} .
\end{aligned}
$$

In this case $\mu_{00}=\mu_{00}^{(a)}+\mu_{00}^{(b)}+\mu_{00}^{(c)}$. The term "symmetric" here means that the formula is equivalent for each channel, if we permute the channels, we obtain the same formula.

We need some common $\mu_{00}$ and centroid of all channels, the simplest unification of that from individual channels is

$$
\begin{aligned}
& m_{00}=m_{00}^{(a)}+m_{00}^{(b)}+\ldots, \\
& x_{c}=\left(m_{10}^{(a)}+m_{10}^{(b)}+\ldots\right) / m_{00}, \quad y_{c}=\left(m_{01}^{(a)}+m_{01}^{(b)}+\ldots\right) / m_{00} .
\end{aligned}
$$

The central moments are centered with respect to the common centroid

$$
\mu_{p q}^{(a)}=\int_{-\infty}^{\infty} \int_{-\infty}^{\infty}\left(x-x_{c}\right)^{p}\left(y-y_{c}\right)^{q} a(x, y) d x d y \quad p, q=0,1,2, \ldots .
$$

Now, channel centroids $x_{c}^{(k)}=m_{10}^{(k)} / m_{00}^{(k)}, y_{c}^{(k)}=m_{01}^{(k)} / m_{00}^{(k)}, k=\mathrm{a}, \mathrm{b}, \ldots$ can differ from the common centroid $x_{c}, y_{c}$. First-order moments need not be zero and we can use them for the construction of additional invariants, e.g.

$$
I_{C 1}^{(a, b)}=\left(\mu_{10}^{(a)} \mu_{01}^{(b)}-\mu_{10}^{(b)} \mu_{01}^{(a)}\right) / \mu_{00}^{3} .
$$

The value is zero, if computed from two channels only, $m_{00}, x_{c}$ and $y_{c}$ (5) must include a certain third channel for a non-zero result. Another example of a simultaneous invariant of first and second orders

$$
I_{C 12}^{(a, b)}=\left(\mu_{20}^{(a)}\left(\mu_{01}^{(b)}\right)^{2}+\mu_{02}^{(a)}\left(\mu_{10}^{(b)}\right)^{2}-2 \mu_{11}^{(a)} \mu_{10}^{(b)} \mu_{01}^{(b)}\right) / \mu_{00}^{5} .
$$

Even zero-order two-channel affine invariant does exist:

$$
I_{C 0}^{(a, b)}=\mu_{00}^{(a)} / \mu_{00}^{(b)} .
$$

The rule concerning the number of invariants goes through the wringer, indices 0,0 really satisfy five constraints at the same time - all except scaling. If we compute affine moment invariants from a color photograph, we can use 12 additional invariants computed from more than one channel. Generally, if we have a multi-channel image with more than three channels, we can use 6 additional invariants per channel. 


\section{Numerical Experiment}

The goal of this experiment is to show properties of the new moment invariants in pattern recognition. We have photographed a series of cards used in a game called mastercards (also pexeso), where the objective is to find the same pairs of cards turned face-down. Cards from each of the ten pairs are shown on Fig. 1.

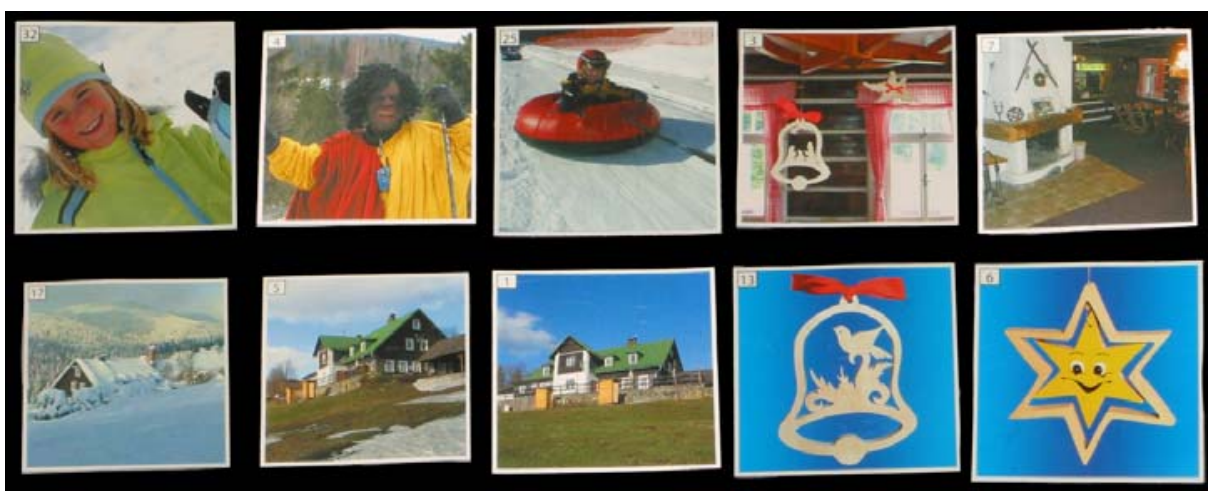

Fig. 1. The mastercards. First row from left: Girl, Old scratch, Tyre-ride, Room-bell and Fireplace, second row: Winter cottage, Spring cottage, Summer cottage, Bell and Star.

Each card was captured eight times, rotated by approximately $45^{\circ}$ between consecutive snaps. An example of the rotation of a pair of cards is shown on Fig. 2. Any small deviation from the perpendicular direction during capturing carried a small projective distortion that can be approximated by the affine transform. The cards were snapped on a dark background and segmented by region growing while small objects (less than 10000 pixels) were removed. The first snap of each card was used as a representative of its class and the following seven snaps were recognized by the minimum-distance classifier, so the theoretical maximum number of errors is 140 .

Our feature set includes $I_{1}, I_{2}, I_{3}, I_{4}$ and $I_{C 12}^{(a, a)}$ from each channel, $I_{C 0}^{(R, G)}$, $I_{C 0}^{(B, G)}, I_{C 1}^{(R, B)}, I_{C 12}^{(R, B)}, I_{C 2}^{(R, G)}$ and $I_{C 2}^{(B, G)}$, i.e. 21 invariants. The complete set should include additional 3 invariants, e.g. $I_{C 23}^{(R, B)}, I_{C 3}^{(R, G)}$ and $I_{C 3}^{(B, G)}$, we omitted them because of the comparison with the same number of invariants from the other set. The moments are always centered to the common center of all 3 channels. The invariants are normalized to magnitude by the following procedure. The moments are first normalized to scaling

$$
\tilde{\mu}_{p q}=\frac{\mu_{p q}}{\mu_{00}^{\frac{p+q}{2}+1}},
$$




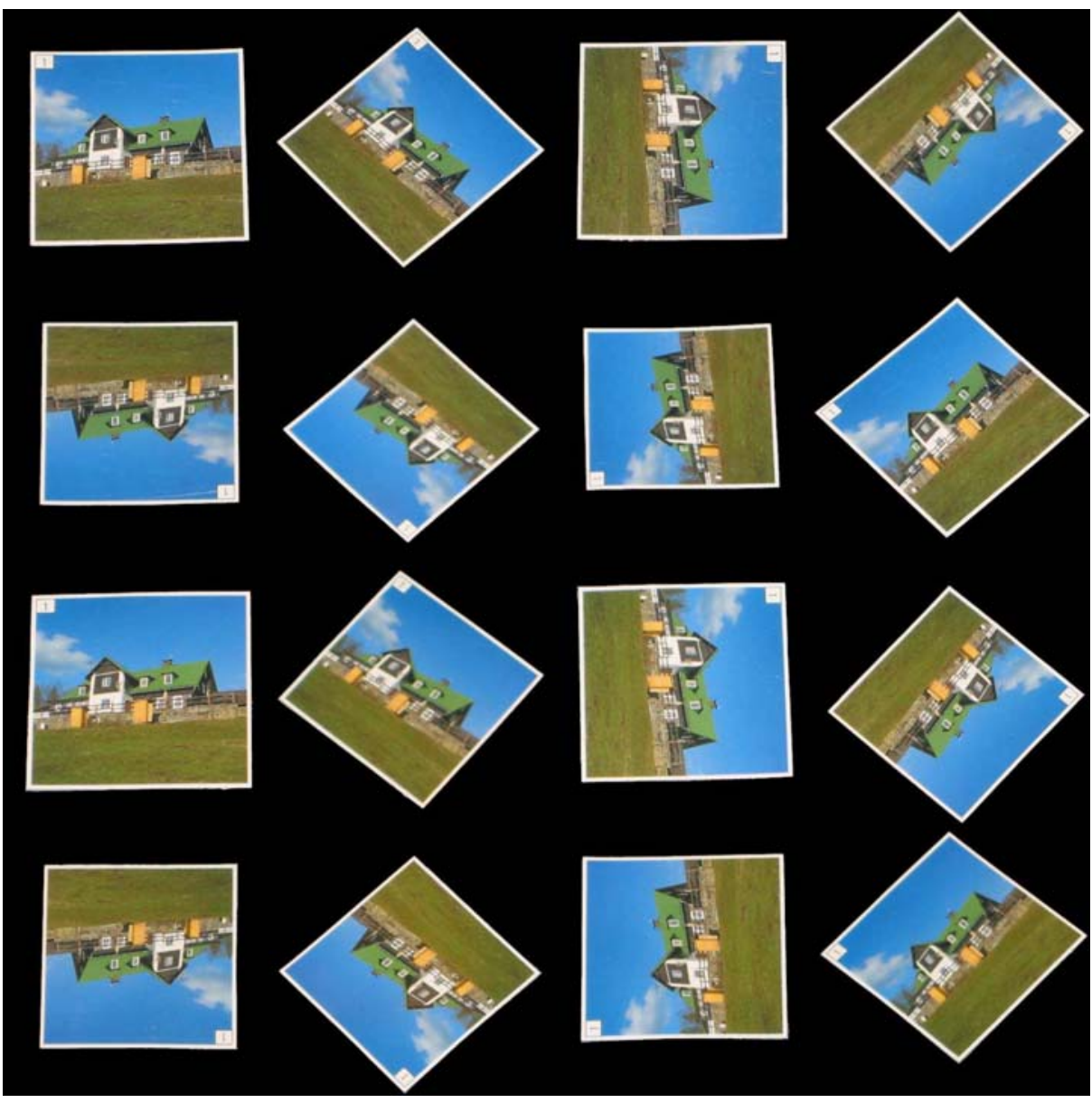

Fig. 2. The card "Summer cottage" with all rotations. The third and fourth rows contain the other card from the pair.

then to the magnitude

$$
\hat{\mu}_{p q}=\pi^{\frac{p+q}{2}}\left(\frac{p+q}{2}+1\right) \tilde{\mu}_{p q},
$$

and then the invariants are computed and normalized to the degree

$$
\hat{I}=\operatorname{sign}(I)|I|^{\frac{1}{r}}
$$

where $r$ is the degree of the invariant, i.e. the number of moments in one term. The minimum distance classifier was used. The cards were classified correctly, without an error. The classification of a card as the other card from the pair was 
not considered an error. Nevertheless, we can see the feature space of the zerothorder invariants on Fig. 3 and some clusters are divided into two subclusters, i.e. the cards from that pair were distinct, while other pairs create compact clusters. Together $102(73 \%)$ cards were assigned to the correct card from the pair, while $38(27 \%)$ cards were assigned to the other card from the pair, but this datum depends not only on the quality of the features, but also on the actual differences of both cards.

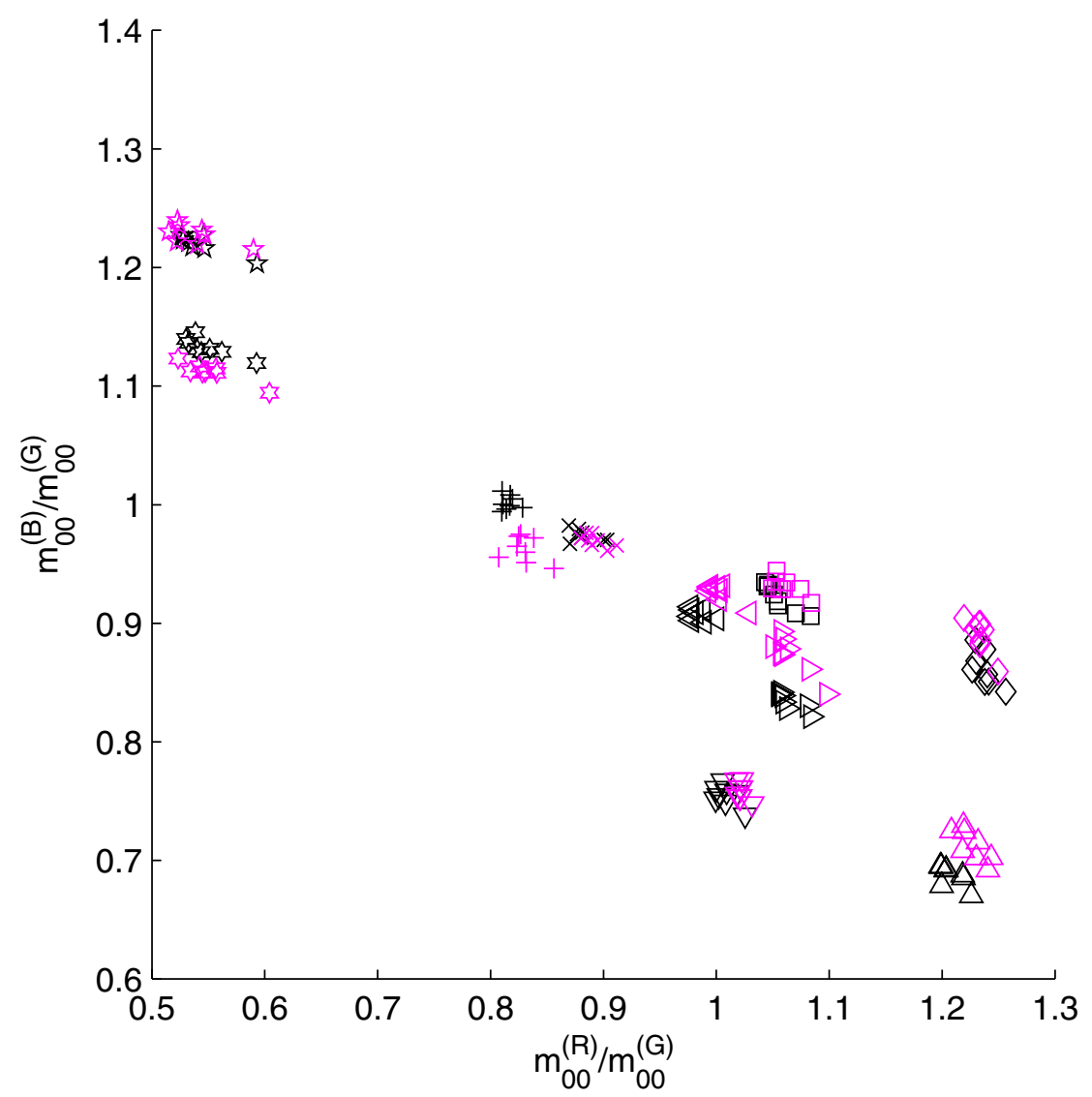

Fig. 3. The mastercards. Legend: $\nabla$ - Girl, $\triangle$ - Old scratch, $\square-$ Tyre-ride, $\diamond-$ Roombell and $\triangleright-$ Fireplace, $\times$ - Winter cottage, $\triangleleft$ - Spring cottage, +- Summer cottage, - Bell and $s$-Star. A card from each pair is expressed by the black symbol while the other card is expressed by the magenta (gray) symbol.

Next, we carried out the experiment with one-channel invariants $I_{1}, I_{2}, I_{3}$ and $I_{4}$ only, i.e. 12 features together, centered to the centroid of each channel separately. The cards were classified with 3 errors $(2.1 \%)$, which means 
the new invariants bring better discriminability and consequently improve the recognition.

For comparison, the same experiment was repeated with the invariants from [2, labeled "GPD invariants" in [4]. The number of invariants was the same as in the first experiment, i.e. 21, they were normalized by the standard deviation through the whole set and provided classification of the objects with 6 errors (4.3\%). This can still be considered a good result, but obviously, new invariants can improve the recognition, when the illumination changes are small.

\section{Conclusion}

A new type of affine moment invariants for multi-channel images was proposed primarily for color images. They yield better results than the current invariants combining affine invariance with invariance to illumination changes. Nevertheless, the new invariants can be normalized to simple contrast changes as well.

The new invariants have a low order and therefore high robustness to noise, and include even zeroth-order invariants, which does not exist in the case of one-channel invariants.

\section{References}

1. Van Gool, E.P.L., Moons, T., Oosterlinck, A.: Vision and lie's approach to invariance. Invited paper for Image and Vision Computing 13, 259-277 (1995)

2. Mindru, F., Moons, T., Van Gool, L.: Color-based moment invariants for viewpoint and illumination independent recognition of planar color patterns. In: International Conference on Advances in Pattern Recognition, ICAPR 1998, pp. 113-122 (1998)

3. Mindru, F., Moons, T., van Gool, L.: Recognizing color patterns irrespective of viewpoint and illumination. In: Proc. IEEE Conf. Computer Vision Pattern Recognition, CVPR 1999, vol. 1, pp. 368-373 (1999)

4. Mindru, F., Tuytelaars, T., Van Gool, L., Moons, T.: Moment invariants for recognition under changing viewpoint and illumination. Computer Vision and Image Understanding 94, 3-27 (2004)

5. Flusser, J., Suk, T.: Pattern recognition by affine moment invariants. Pattern Recognition 26, 167-174 (1993)

6. Suk, T., Flusser, J.: Graph method for generating affine moment invariants. In: ICPR 2004, 17th International Conference on Pattern Recognition, pp. 192-195. IEEE Computer Society, Los Alamitos (2004)

7. Suk, T., Flusser, J.: Tables of affine moment invariants generated by the graph method. Research Report 2156, Institute of Information Theory and Automation (2005)

8. Hilbert, D.: Theory of Algebraic Invariants. Cambridge University Press, Cambridge (1993) 